\title{
The Role of T Cell Help for Anti-viral CTL Responses
}

\author{
Dominik Wodarz* ${ }^{*}$ and Vincent A. A. Jansen $\$$ \\ *Institute for Advanced Study, Olden Lane, Princeton, NJ 08540, U.S.A. and \\ $\ddagger$ School of Biological Sciences, Royal Holloway, University of London, Egham, Surrey TW20 0EX, U.K.
}

(Received on 29 August 2000; Accepted in revised form on 17 May 2001)

\begin{abstract}
Cytotoxic T lymphocyte (CTL or CD8) responses are a major branch of the immune system involved in controlling viral infections. Murine models have shown that the development of effective and sustained CD8 cell responses requires CD4 T cell help. However, the precise mechanism in which CD4 cells provide help for CD8 cell responses is still controversial. In the literature, mainly two mechanisms are discussed. According to the "classical" pathway, CD4 cells secrete cytokines, such as IL-2, which promote the responsiveness of the CD8 cells. According to the "CD4-APC-CD8" pathway, CD4 cells specifically activate antigen presenting cells (APCs), and APCs specifically interact with CD8 cells, thereby delivering help. Here, we derive kinetic models in order to describe and compare both pathways of help. We find that the two pathways might have different roles in different situations. The classical pathway is more efficient at inducing CD8 cell expansion at high virus loads, while the CD4-APC-CD8 pathway is more efficient at inducing CD8 cell proliferation at low virus loads. From this, it follows that the classical pathway might be needed in order to kick-start a CD8 cell response in the acute phase of the infection, while the CD4-APC-CD8 pathway is needed in order to ensure virus clearance when virus load is reduced by the immune system. These findings have implications for the interpretation of experimental data from virus infection in helper-deficient hosts. In particular, the models offer further suggestions for the development of treatment regimes aimed at achieving immunological control of HIV infection which has been shown to crucially depend on the availability of helper cell responses.
\end{abstract}

(C) 2001 Academic Press

\section{Introduction}

The CD4 T cell population is a central component of the immune system responsible for orchestrating specific immune responses. Although $\mathrm{T}$ helper cells can have effector function themselves and directly inhibit viral replication (Christensen et al., 1999), their main role is to provide help for B cell and cytotoxic $\mathrm{T}$ cell responses (CTL). B cells secrete antibodies which can inhibit free virus particles, while CTL lyse

\footnotetext{
$\dagger$ Author to whom correspondence should be addressed. E-mail: wodarz@ias.edu
}

infected cells or secrete chemokines inhibiting viral replication. The CTL response is thought to be a major component of the immune system responsible for fighting viral infections (Jeffery et al., 1999; Kagi et al., 1996; Schmitz et al., 1999). Absence of CD4 T cell help can result in impaired anti-viral CTL responses, and renders the immune system unable to control viral replication in the long term. This has been studied extensively with murine LCMV infection. Although the primary CTL response in class II knockout mice is similar to that observed in wild-type mice, the absence of CD4 cell help can result in strongly impaired CTL responses in the memory phase 
(a)

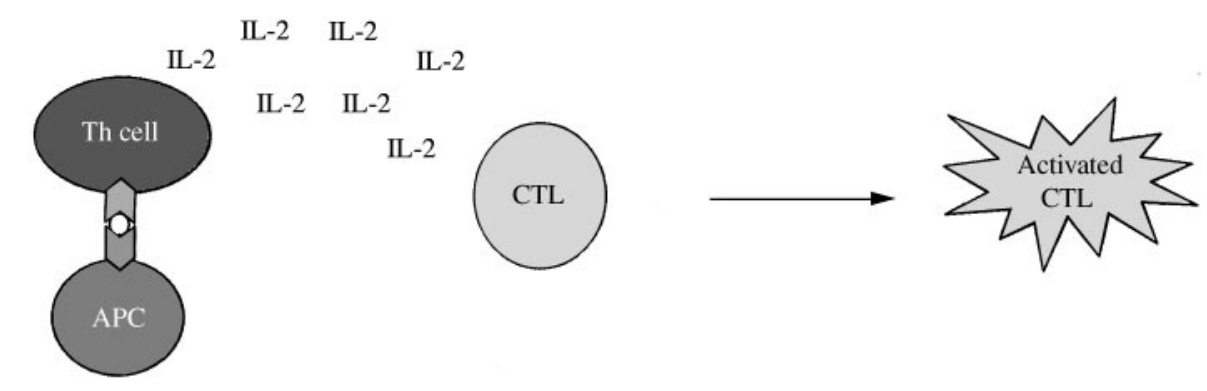

(b)

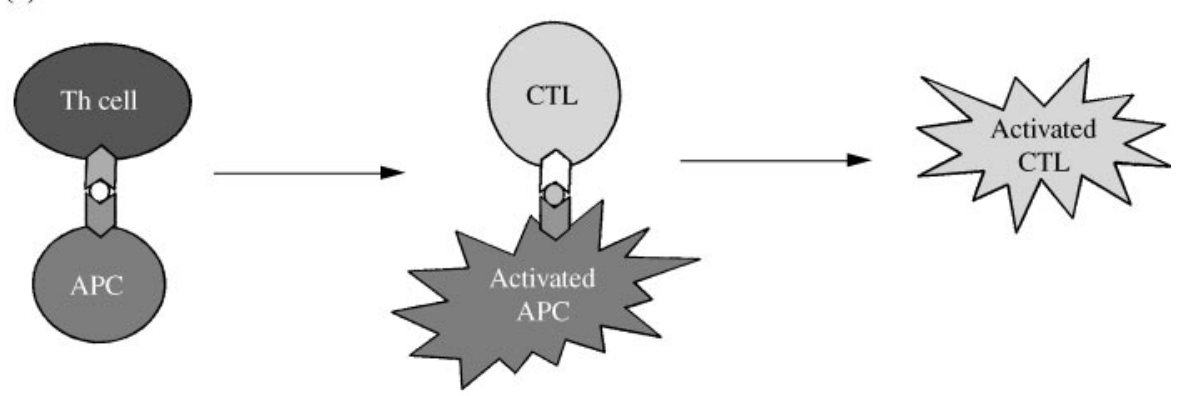

FIG. 1. Schematic diagram of the two pathways of help explored in this paper. (a) According to the classical pathway, CD4 $\mathrm{T}$ cells secrete IL-2 which promotes proliferation of CD8 cells. (b) According to the CD4-APC-CD8 pathway, CD4 cells activate APCs which in turn specifically interact with CD8 cells, delivering help.

of the infection, accompanied by uncontrolled viral growth (Andreasen et al., 2000; Borrow et al., 1996, 1998; Thomsen et al., 1996, 1998). Viruses with a tropism for $\mathrm{T}$ helper cells can potentially impair the CD4 cell response, resulting in compromised CTL and persistent infection. This seems to be the case with HIV (Kalams \& Walker, 1998; Rosenberg et al., 1997). In most patients, already the acute phase is characterized by the marked absence of significant levels of specific CD4 cell proliferative responses and relatively weak CTL activity (Lifson et al., 2000; Rosenberg et al., 2000). On the other hand, longterm non-progressors are characterized by the presence of significant levels of CD4 cell proliferative responses and show high levels of CTL despite extremely low viral loads (Harrer et al., 1996a, b). The precise mechanisms underlying CD4 cell help for CD8 cell responses are still unclear. Traditionally, the main role of CD4 T cells was thought to be the secretion of cytokines upon contact with antigen. These cytokines, especially IL-2, are thought to stimulate CD8 cells, promoting their expansion and maintenance. This mechanism will be referred to as the "classical pathway" [Fig. 1(a)]. However, recently, it has become clear that a more complicated pathway might be involved in the generation of help for CD8 cell responses (Ridge et al., 1998; Schoenberger et al., 1998). Experiments suggest that CD4 $\mathrm{T}$ cells can specifically interact with antigen presenting cells (APC), thereby activating them. Activated APCs in turn can specifically interact with CD8 T cells, delivering help in the form of costimulatory signals. We will refer to this mechanism as the CD4-APC-CD8 pathway [Fig. 1(b)]. A better understanding of the mechanisms underlying help, as well as the relative roles of these different pathways are important not only from a conceptual point of view, but also in the context of vaccination and immuno-therapy. From this outline it is clear that the mechanisms underlying CD4 cell help for CD8 cell responses are complex and involve many different components. Hence, mathematical models can help us to improve our understanding of these processes (De Boer \& Perelson, 1994). We formulate kinetic models describing 
potential interactions involved in help for CD8 cell responses. We start by deriving kinetic models for the CD4-APC-CD8 pathway, and compare this to a model describing the classical pathway of help. We find that both pathways could be important in different situations. The CD4-APC-CD8 pathway induces efficient CTL expansion at low virus loads, while the classical pathway more efficiently induces CD8 cell responses at high virus loads. We discuss the significance of these findings in relation to different stages of the infectious process, and interpret data on virus infections in helper-deficient hosts. Finally, the models suggest an experimental test in order to evaluate the role of the two pathways in viral infections.

\section{The CD4-APC-CD8 Pathway}

According to the CD4-APC-CD8 pathway, CD4 T cells specifically activate APCs, and activated APCs then specifically interact with CD8 cells, promoting their expansion. This is mediated by receptor-ligand interactions, such as CD40-CD40L or B7-CD28 (Borrow et al., 1998; Thomsen et al., 1998; Andreasen et al., 2000). Thus, the CD4-APC-CD8 pathway can be represented by the following reaction scheme:

$$
\begin{aligned}
& \mathrm{Th}+\mathrm{A} \underset{k_{2}}{\stackrel{k_{1}}{\rightleftharpoons}} \mathrm{ThA}, \\
& \mathrm{ThA} \stackrel{k_{3}}{\rightarrow} \mathrm{Th}+\mathrm{A}^{*}, \\
& \mathrm{CD} 8+\mathrm{A} * \underset{k_{5}}{\stackrel{k_{4}}{\rightleftharpoons}} \mathrm{CD} 8 \mathrm{~A}^{*}, \\
& \mathrm{CD} \mathrm{A}^{*} \stackrel{k_{6}}{\rightarrow}(n+1) \mathrm{CD} 8+\mathrm{A}^{*}, \\
& \mathrm{~A}^{*} \stackrel{k_{7}}{\rightarrow} A,
\end{aligned}
$$

where Th stands for helper cells, A stands for APC, and a star represents an activated state. Every CD8 cell that binds to an activated APC and that itself will get activated will through proliferation give rise to $n$ new CD8 cells. Note that in assuming this we have aggregated CD8 activation and proliferation in one process. The $k_{i}$ are reaction constants. It is assumed that the number of APCs is small and that this limits the reactions. The kinetics of these reactions are given by

$$
\begin{aligned}
& \frac{\mathrm{d}[\mathrm{A}]}{\mathrm{d} t}=-k_{1}[\mathrm{~A}][\mathrm{Th}]+k_{2}[\mathrm{ThA}]+k_{7}\left[\mathrm{~A}^{*}\right], \\
& \frac{\mathrm{d}\left[\mathrm{A}^{*}\right]}{\mathrm{d} t}=k_{3}[\mathrm{ThA}]-k_{4}\left[\mathrm{~A}^{*}\right][\mathrm{CD} 8] \\
& +\left(k_{5}+k_{6}\right)\left[\mathrm{CD}^{2} \mathrm{~A}^{*}\right]-k_{7}\left[\mathrm{~A}^{*}\right] \\
& \frac{\mathrm{d}[\mathrm{Th}]}{\mathrm{d} t}=\left(k_{2}+k_{3}\right)[\mathrm{ThA}]-k_{1}[\mathrm{~A}][\mathrm{Th}], \\
& \frac{\mathrm{d}[\mathrm{CD} 8]}{\mathrm{d} t}=-k_{4}\left[\mathrm{~A}^{*}\right][\mathrm{CD} 8] \\
& +\left(k_{5}+(n+1) k_{6}\right)\left[\mathrm{CD} 8 \mathrm{~A}^{*}\right], \\
& \frac{\mathrm{d}[\mathrm{ThA}]}{\mathrm{d} t}=k_{1}[\mathrm{~A}][\mathrm{Th}]-\left(k_{2}+k_{3}\right)[\mathrm{ThA}], \\
& \frac{\mathrm{d}\left[\mathrm{CD} 8 \mathrm{~A}^{*}\right]}{\mathrm{d} t}=k_{4}\left[\mathrm{~A}^{*}\right][\mathrm{CD} 8] \\
& -\left(k_{5}+k_{6}\right)\left[\mathrm{CD} 8 \mathrm{~A}^{*}\right]
\end{aligned}
$$

where the square brackets denote concentrations of cell types or complexes of cells. We next assume that the kinetics of complexes are fast compared to the other reactions. This amounts to the assumptions that $k_{2}+k_{3} \gg k_{1}$ and $k_{5}+k_{6} \gg k_{4}$. Under these assumptions both complexes go to their quasi-steady state and the concentration will approximately be $[\mathrm{ThA}]=$ $\left(k_{1} /\left(k_{2}+k_{3}\right)\right)[\mathrm{Th}][\mathrm{A}]$ and $\left[\mathrm{CD} 8 \mathrm{~A}^{*}\right]=\left(k_{4} /\left(k_{5}+\right.\right.$ $\left.k_{6}\right)$ ) $\left[\mathrm{A}^{*}\right][\mathrm{CD} 8]$. This reduces the kinetics to

$$
\begin{aligned}
& \frac{\mathrm{d}[\mathrm{A}]}{\mathrm{d} t}=-\frac{k_{1} k_{3} x}{k_{2}+k_{3}}[\mathrm{~A}]+k_{7}\left[\mathrm{~A}^{*}\right], \\
& \frac{\mathrm{d}\left[\mathrm{A}^{*}\right]}{\mathrm{d} t}=\frac{k_{1} k_{3} x}{k_{2}+k_{3}}[\mathrm{~A}]-k_{7}\left[\mathrm{~A}^{*}\right], \\
& \frac{\mathrm{d}[\mathrm{CD} 8]}{\mathrm{d} t}=n \frac{k_{4} k_{6}}{k_{5}+k_{6}}\left[\mathrm{~A}^{*}\right][\mathrm{CD} 8] .
\end{aligned}
$$


Note that the concentration of free helper cells is constant under these assumptions. Let the total number of helper cells be given by $x=$ $[\mathrm{Th}]+[\mathrm{ThA}]$. Under the assumptions made, the number of helper cells in complexes is small compared to the number of free helper cells. We can therefore assume that $[\mathrm{Th}]=x$. The total number of APCs is given by $y=[\mathrm{A}]+\left[\mathrm{A}^{*}\right]+$ $\left(k_{1} x /\left(k_{2}+k_{3}\right)\right)[\mathrm{A}]+\left(k_{4} /\left(k_{5}+k_{6}\right)\right)[\mathrm{A} *][\mathrm{CD} 8]$. Note that the number of free APCs, $[\mathrm{A}]+\left[\mathrm{A}^{*}\right]$, is constant and the ratio of free activated to free non-activated APCs will equilibrate. At equilibrium we find

$\left[\mathrm{A}^{*}\right]$

$$
\begin{aligned}
& =\frac{k_{1} k_{3} x y}{k_{7}\left(k_{2}+k_{3}\right)+k_{1} k_{3} x\left(1+\left(k_{4} /\left(k_{5}+k_{6}\right)\right)[\mathrm{CD} 8]\right)+k_{1} k_{7} x} \\
& =\frac{\varepsilon x y}{1+\varepsilon x\left(1+f_{c} z\right)+f_{h} x},
\end{aligned}
$$

where $\varepsilon=k_{1} k_{3} / k_{7}\left(k_{2}+k_{3}\right)$ is the net reaction constant of APC activation, $c=n k_{4} k_{6} /\left(k_{5}+k_{6}\right)$ is the net reaction constant of CD8 activation, $f_{h}=k_{1} /\left(k_{2}+k_{3}\right)$ is a proportionality constant for the Th-APC complex, and $f_{c}=k_{4} /\left(k_{5}+k_{6}\right)$ is a proportionality constant for the CD8-APC complex.

We denote the number of CD8 $\mathrm{T}$ cells by $z=[\mathrm{CD} 8]+\left[\mathrm{CD} 8 \mathrm{~A}^{*}\right]$. Under the assumption that the number of CD8 cells in complexes is negligible compared to the total number, we can assume that $[\mathrm{CD} 8]=z$. The proliferation of these cells is described by

$$
\frac{c \varepsilon x y z}{1+\varepsilon x\left(1+f_{c} z\right)+f_{h} x} .
$$

This is a form of the proliferation function that generalizes several functions which have been described previously. For instance, by assuming that the amount of help, $x$ is constant we find

$$
\frac{\alpha y}{\beta+z} z
$$

where $\alpha=c / f_{c}, \beta=1 /\left(\varepsilon x f_{c}\right)+\left(\varepsilon+f_{h}\right) /\left(\varepsilon f_{c}\right)$. This proliferation function has been derived by Fishman \& Perelson (1993), De Boer \& Perelson
(1994) and Borghans et al. (1999). Another possible assumption is that the help is vanishingly small, which amounts to linearizing the proliferation function in $x$ in the neighbourhood of $x=0$, which results in

$$
\operatorname{cexyz}
$$

which is arguably the simplest possible functional form (Nowak \& Bangham, 1996; Wodarz \& Nowak, 1999). In the derivation of the general proliferation function it was assumed that $k_{2}+k_{3} \gg k_{1}$ and $k_{5}+k_{6} \gg k_{4}$. Hence, it is natural to assume that $f_{h}$ and $f_{c}$ can be ignored, which results in

$$
\frac{c \varepsilon x y z}{1+\varepsilon x}
$$

This functional form has also been used to describe CTL dynamics (Wodarz et al., 1999; Wodarz \& Nowak, 2000). In the rest of this paper we will use this particular functional form. The rate of CTL activation and proliferation is thus a saturating function of the number of helper cells with $1 / \varepsilon$ as the half saturation constant. In the presence of large amounts of help, the rate of CTL proliferation approximates $c y z$, where the parameter $c$ denotes the CTL responsiveness. This parameter includes a variety of factors which influence the ability of the CTL to respond to antigen, determined by the $\mathrm{T}$ cell receptor and the MHC type of the host. The level of help is given by the number of helper cells, $x$, as well as the efficacy of those helper cells, $\varepsilon$. The higher the efficacy of help, $\varepsilon$, the lower the number of helper cells, $x$, required to induce maximal stimulation of the CTL response.

If we then assume that CD8 cells die with a rate $b z$ we get the kinetics

$$
\frac{\mathrm{d} z}{\mathrm{~d} t}=\frac{c \varepsilon x y z}{1+\varepsilon x}-b z
$$

In this model, the CTL response becomes established if $\varepsilon x>b /(c \tilde{y}-b)$, where $\tilde{y}$ denotes virus load in the absence of a CTL response. In other words, the CTL response only becomes established if the amount of CD4 cell help lies above 

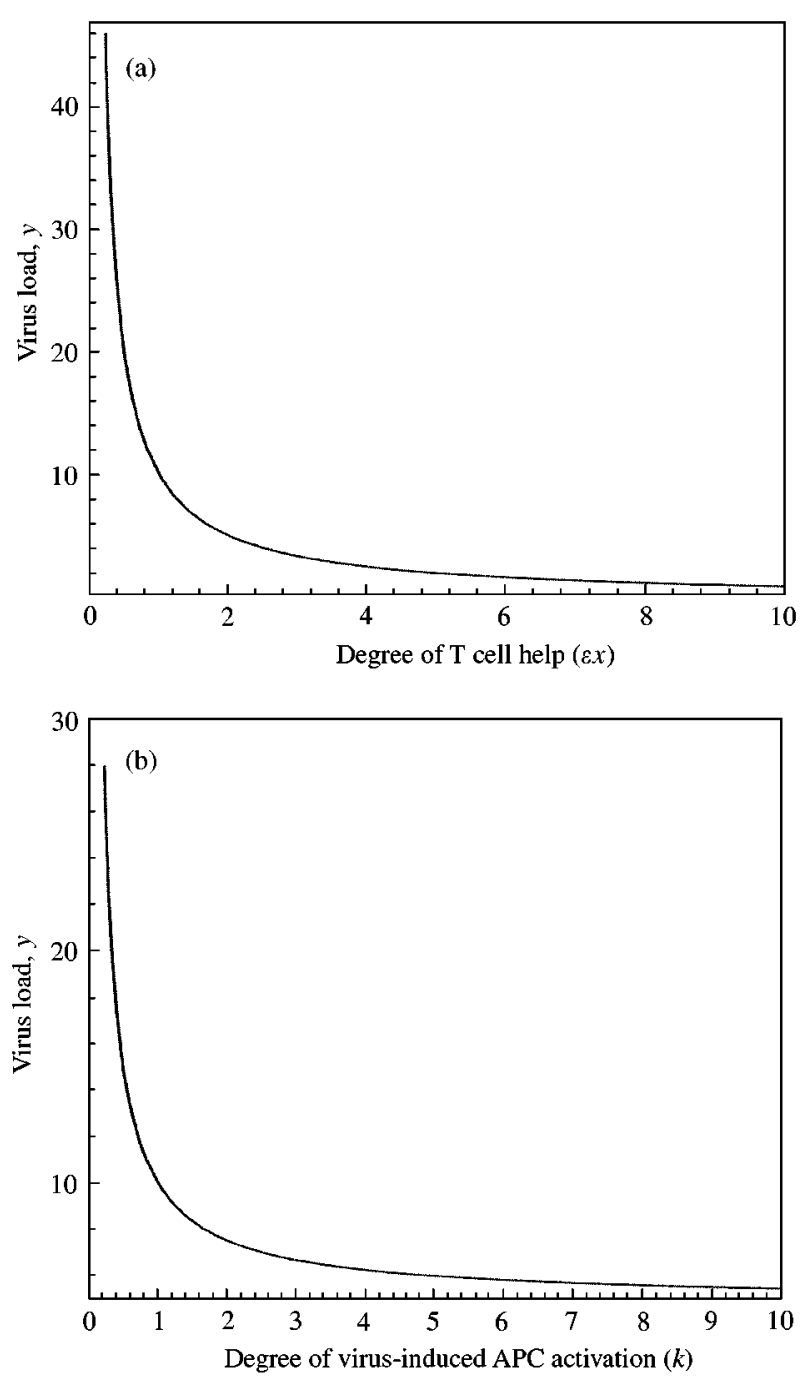

FIG. 2. The effect of help on virus load. (a) Virus load decreases asymptotically with increasing degrees of help. Virus load approaches a value of $b / c$ for high degrees of help. (b) Increasing the rate of virus-induced APC activation also results in an asymptotic decrease in virus load. For large values of $k$, virus load approaches a value of $b / c$. Parameter values were chosen as follows. (a) $c=1 ; b=0.1 ; \varepsilon=0.01$; (b) $c=0.1 ; b=0.5 ; \varepsilon=0.01 ; x=0$.

a critical threshold. This result makes sense in that an immune response is wasted when triggered by too low concentrations of antigen. Equilibrium virus load in the presence of CTL is given by $\hat{y}=b(1+\varepsilon \hat{x}) / c \varepsilon \hat{x}$. As shown in Fig. 2, an increase in the amount of help reduces virus load down to an asymptote, at which the helperinduced stimulation of the CTL response has reached its maximum. At the asymptote, $\hat{y}=b / c$.
The equilibrium viral load is determined by other immunological processes. Within the framework presented here we can model the proliferation of $\mathrm{T}$ helper cells in response to activation by APCs. If we assume that $T$ cell proliferation is proportional to the amount of $\mathrm{T}$ cells in complexes we find that $\mathrm{T}$ cell proliferation is given by $\phi x y$, where $\phi$ is the proliferation rate. If the death rate of $\mathrm{T}$ cells is given by $\psi$ we can write for the $\mathrm{T}$ cell dynamics

$$
\frac{\mathrm{d} x}{\mathrm{~d} t}=\phi x y-\psi x
$$

\section{Direct Activation}

Although the presence of CD4 + T cells is required for efficient generation of CTL responses in many virus infections, experiments suggest that APCs could be stimulated and activated directly without the need for CD4 T cells. It is thought that direct activation of APCs can be induced experimentally by crosslinking of CD40 on the APCs (Ridge et al., 1998; Schoenberger et al., 1998). In vivo, experiments suggest that viral infection of APCs has a similar effect (Andreasen et al., 2000). We can modify the above model to account for a CD4 cell-independent component of the CTL response.

If APCs can also become stimulated and activated directly by the virus without the need for $\mathrm{T}$ helper cells, the following reaction should be appended to the reaction scheme (1):

$$
\mathrm{A} \stackrel{k_{8}}{\rightarrow} \mathrm{A}^{*}
$$

where $k_{8}$ is a reaction constant. After making the same quasi-steady-state assumptions as in the previous section, this changes the kinetics of the APCs to

$$
\begin{gathered}
\frac{\mathrm{d}[\mathrm{A}]}{\mathrm{d} t}=-\frac{k_{1} k_{3} x}{k_{2}+k_{3}}[\mathrm{~A}]+k_{7}\left[\mathrm{~A}^{*}\right]-k_{8}[\mathrm{~A}], \\
\frac{\mathrm{d}\left[\mathrm{A}^{*}\right]}{\mathrm{d} t}=\frac{k_{1} k_{3} x}{k_{2}+k_{3}}[\mathrm{~A}]-k_{7}\left[\mathrm{~A}^{*}\right]+k_{8}[\mathrm{~A}] .
\end{gathered}
$$


The total number of APCs, $y$, is preserved and the number of activated APCs will equilibrate to

$$
\left[\mathrm{A}^{*}\right]=\frac{(\varepsilon x+k) y}{1+(\varepsilon x+k)\left(1+f_{c} z\right)+f_{h} x},
$$

where $k=k_{8} / k_{7}$. By ignoring the terms contain$\operatorname{ing} f_{c}$ and $f_{h}$ this reduces to

$$
\left[\mathrm{A}^{*}\right]=\frac{(\varepsilon x+k) y}{1+\varepsilon x+k} \text {. }
$$

The proliferation rate of $\mathrm{T}$ cells in this case becomes $c(\varepsilon x+k) y /(1+\varepsilon x+k)$. The parameter $k$ represents the rate of helper-independent activation of APC. This description does not rely on the detailed mechanism by which helperindependent expansion of the CTL population is achieved, a topic which is still open to debate (Andreasen et al., 2000). The effect of direct stimulation is effectively that $k / \varepsilon$ less CD 4 cells are needed in order to achieve the same amount of help. In the model, a sustained CTL response becomes established if $x+k / \varepsilon>b / \varepsilon(c \tilde{y}-b)$, where $\tilde{y}$ stands for the level of antigen in the absence of CTL. In other words, even if CD4 cells are absent, the CTL response can still invade if the rate of helper-independent activation of APC, $k$, is sufficiently high. Equilibrium virus load in the presence of CTL is given by $\hat{y}=b(1+$ $\varepsilon \hat{x}+k) / c(\varepsilon \hat{x}+k)$. As shown in Fig. 2(b), higher rates of virus-induced activation of APCs reduce virus load down to an asymptote at which $\hat{y}=b / c$.

\section{THE CLASSICAL PATHWAY}

For the classical pathway of $\mathrm{T}$ cell proliferation it is assumed that CD8 $\mathrm{T}$ cells require contact with the antigen and exposure to cytokines, denoted as I, in order to proliferate. The cytokines are released by CD4 cells upon contact with antigen. This amounts to the following reaction scheme:

$$
\begin{gathered}
\mathrm{Th}+\mathrm{A}^{*} \underset{k_{1}}{\stackrel{k_{2}}{\rightleftharpoons}} \mathrm{ThA}, \\
\mathrm{ThA} \stackrel{k_{3}}{\rightarrow} \mathrm{Th}+\mathrm{A}+\mathrm{I}, \\
\mathrm{CD} 8+\mathrm{A} \underset{k_{4}}{\stackrel{k_{5}}{\rightleftharpoons}} \mathrm{CD} 8 \mathrm{~A},
\end{gathered}
$$

$$
\mathrm{CD} 8 \mathrm{~A}+\mathrm{I} \stackrel{k_{6}}{\rightarrow}(n+1) \mathrm{CD} 8+\mathrm{A}+\mathrm{I},
$$

$$
\stackrel{k_{9}}{\rightarrow}
$$

with kinetics:

$$
\begin{aligned}
\frac{\mathrm{d}[\mathrm{A}]}{\mathrm{d} t}= & -k_{1}[\mathrm{Th}][\mathrm{A}]+\left(k_{2}+k_{3}\right)[\mathrm{ThA}] \\
& -k_{4}[\mathrm{CD} 8][\mathrm{A}]+\left(k_{5}+k_{6}[\mathrm{I}]\right)[\mathrm{CD} 8 \mathrm{~A}], \\
\frac{\mathrm{d}[\mathrm{Th}]}{\mathrm{d} t}= & -k_{1}[\mathrm{Th}][\mathrm{A}]+\left(k_{2}+k_{3}\right)[\mathrm{ThA}], \\
\frac{\mathrm{d}[\mathrm{CD} 8]}{\mathrm{d} t}= & -k_{4}[\mathrm{CD} 8][\mathrm{A}] \\
& +\left(k_{5}+(n+1) k_{6}[\mathrm{I}]\right)[\mathrm{CD} 8 \mathrm{~A}], \\
\frac{\mathrm{d}[\mathrm{I}]}{\mathrm{d} t}= & k_{3}[\mathrm{Th}][\mathrm{A}]-k_{9}[\mathrm{I}], \\
\frac{\mathrm{d}[\mathrm{CD} 8 \mathrm{~A}]}{\mathrm{d} t}= & k_{4}[\mathrm{CD} 8][\mathrm{A}]-\left(k_{5}+k_{6}[\mathrm{I}]\right)[\mathrm{CD} 8 \mathrm{~A}], \\
\frac{\mathrm{d}[\mathrm{ThA}]}{\mathrm{d} t}= & k_{1}[\mathrm{Th}][\mathrm{A}]-\left(k_{2}+k_{3}\right)[\mathrm{ThA}] .
\end{aligned}
$$

Under the assumption that $k_{2}+k_{3} \gg k_{1}$ and $k_{5}+k_{6}[\mathrm{I}] \gg k_{4}$ we can also assume that the complexes CD8A and ThA go to their quasisteady states: $[\mathrm{CD} 8 \mathrm{~A}]=\left(k_{4} /\left(k_{5}+k_{6}[\mathrm{I}]\right)\right)$ [CD8][A], and [ThA] $=\left(k_{1} /\left(k_{2}+k_{3}\right)\right)[\mathrm{Th}][\mathrm{A}]$. Under these assumptions the free Th, denoted as $x$, and APC, denoted as $y$, populations are constant (note that this tacitly assumes that the number of Ths and APCs in complexes is neglegible compared to the number of free cells). The kinetics simplify to

$$
\begin{aligned}
\frac{\mathrm{d}[\mathrm{CD} 8]}{\mathrm{d} t} & =\frac{n k_{4} k_{6}[\mathrm{I}]}{k_{5}+k_{6}[\mathrm{I}]}[\mathrm{CD} 8] y, \\
\frac{\mathrm{d}[\mathrm{I}]}{\mathrm{d} t} & =k_{3} x y-k_{9}[\mathrm{I}] .
\end{aligned}
$$




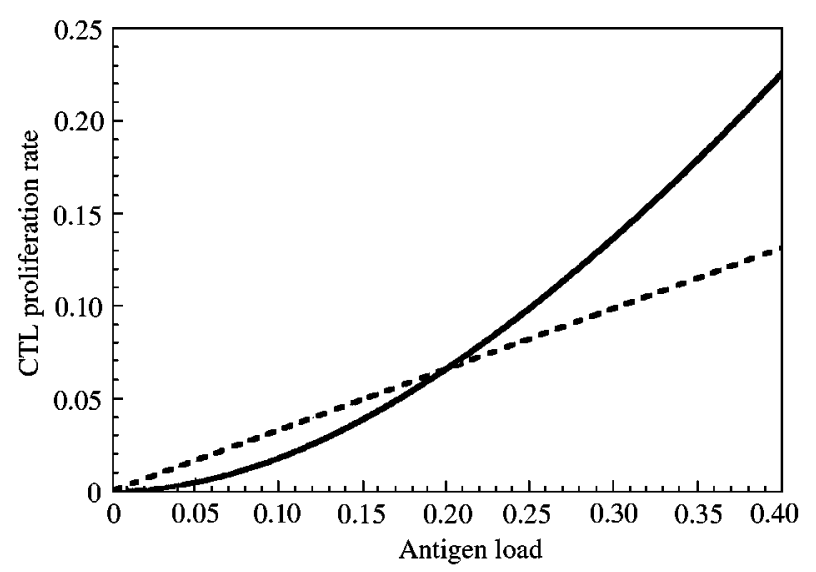

FIG. 3. The rate of CTL proliferation as a function of virus load. The relationship is linear for the CD4APC-CD8 pathway, while it is not for the classical pathway. The CD4-APC-CD8 pathway results in more efficient CD8 cell proliferation at low virus loads. The classical pathway results in more efficient $\mathrm{CD} 8$ cell proliferation at high virus loads. Parameters were chosen as follows: $c=1, \varepsilon=0.5$, $\gamma=2, x=1, \eta=1$ : (- - -) CD4-APC-CD8 pathway; (-) classical pathway.

If, in addition, we assume that the cytokine concentration goes to equilibrium, then by denoting $[\mathrm{CD} 8]=z$ we get the proliferation rate

$$
\frac{\gamma x y^{2} z}{1+\eta x y}
$$

where $\gamma=n k_{4} k_{6} k_{3} / k_{5} k_{9}$ and $\eta=k_{6} k_{3} / k_{5} k_{9}$. According to this expression, the efficacy of such a response depends on the amount of antigenic stimulation. If the antigen concentration is large the proliferation function goes to $(\gamma / \eta) y z$ and the effect of help disappears. In other words, the response is likely to be effective at high virus loads. However, for low antigen concentrations we find $\gamma x y^{2} z$. For low help we recover the same expression. Note that this differs from the proliferation function derived before. Under the classical pathway the proliferation is much lower for low amounts of antigen and an immune response will be slow. The $\mathrm{T}$ helper cell proliferation will not be affected by this different pathway.

The kinetics of the classical pathway are compared to those of the CD4-APC-CD8 pathway in Fig. 3, plotting the rate of CTL proliferation as a function of antigen concentration. While the rate of CTL proliferation is a linear function of antigen concentration under the CD4-APCCD8 pathway, the relationship is not linear under the classical pathway. The classical pathway induces CTL proliferation more efficiently at high antigen concentrations, while the CD4APC-CD8 pathway induces CTL proliferation more efficiently at low antigen concentrations (Fig. 3). This observation follows from the derivation of the proliferation responses. The maximum response under the CD4-APC-CD8 pathway is $c y$, while under the classical pathway the maximum response is given by $\gamma / \eta y$. Since $c=n k_{4} k_{6} /\left(k_{5}+k_{6}\right)<n k_{4}=\gamma / \eta$ the maximum response of the classical pathway exceeds that of the CD4-APC-CD8 pathway.

\section{Application and Discussion}

THE MEANING OF HELP

It is clear that CD4 T cell help plays an important role in the generation of antigen-specific immune responses. The exact mechanism by which help is delivered is, however, still unclear. In this paper, we used mathematical models in order to analyse the role of help in the generation of specific CD8 $\mathrm{T}$ cell responses. We investigated two mechanisms of help which were introduced in the literature. According to the "classical" pathway, CD4 cells secrete cytokines such as IL-2 which bind to and activate specific CD8 cells when exposed to antigen. According to the "CD4-APC-CD8" pathway, the CD4 cells specifically interact with and activate APCs. The activated APCs in turn specifically interact with the CD8 cells and thereby deliver the necessary help. There is evidence supporting a potential role for both of these mechanisms. Experiments suggest that in the absence of any form of help, CD8 T cells become anergic within days following antigenic challenge, not being able to sustain their own proliferation (Deeths et al., 1999). However, exogenous administration of IL-2 resulted in stimulation and proliferation of the CD8 T cells (Deeths et al., 1999). On the other hand, experiments also suggest that help can be administered directly by antigen presenting cells in the absence of CD4 cells. CD4 cells are thought to activate APCs through specific receptor-ligand interactions, such as CD40 and 
CD40L. Crosslinking of CD40 on APCs results in the delivery of help without the need for CD4 cells (Ridge et al., 1998; Schoenberger et al., 1998). With these findings in mind, questions arise regarding the exact nature of help and the relative roles of these two pathways. In this respect, our modelling approach offers new insights. The models suggest that the two pathways of help might both be required in different situations (Fig. 3). At high virus loads, the rate of CD8 cell expansion is faster with the classical pathway compared to the CD4-APC-CD8 pathway (Fig. 3). On the other hand, at low loads, the rate of CD8 cell expansion is slower with the classical pathway compared to the CD4-APC-CD8 pathway (Fig. 3). This could translate to the following situation during the infectious process. At the beginning of the infectious process, during the acute phase, the virus may replicate to relatively high levels before the immune responses kick in and start fighting the pathogen. Hence, the host is faced with the need for specific CD8 cells to expand quickly in response to a relatively high antigenic load. In this situation, the classical pathway of help would be required to generate the most efficient response which could quickly reduce virus load. In this context it is interesting that CD8 cells seem to be able to generate "selfhelp" for a limited period of time by secreting IL-2 themselves (Deeths et al., 1999). This could increase the capacity of the specific CD8 cell response to immediately expand in response to an antigenic challenge. After a limited number of cell divisions, the CD8 cells seem to lose the ability to produce IL-2 themselves, and depend on IL-2 secretion from other immune branches, such as CD4 helper cells (Deeths et al., 1999). Following the primary immune response, virus load starts to decline. Now a parameter region is reached in which efficient immunological pressure at low virus loads is required in order to ensure a continued decline of the virus population towards extinction. In this situation the classical pathway is unlikely to be sufficient, since it results in slow $\mathrm{CD} 8$ cell dynamics at low loads. Instead, the CD4-APC-CD8 pathway will become important to ensure efficient CD8 cell mediated immunity at low loads, and thus to ensure extinction of the virus population. If the CD4-APC-CD8 pathway is not available, modelling suggests that resolution of the infection is likely to be incomplete: although virus load is initially reduced to low levels, immunological pressure is lost at low loads, resulting in the ability of the virus population to grow back and establish a persistent infection.

These verbal arguments can be supported by a simple model, taking both pathways of $\mathrm{T}$ cell help into account. The model is given by the following set of differential equations.

$$
\begin{aligned}
\mathrm{d} x / \mathrm{d} t & =\phi x y-\psi x \\
\mathrm{~d} y / \mathrm{d} t & =r y(1-y / \kappa)-p y z \\
\mathrm{~d} z / \mathrm{d} t= & (c \varepsilon x+k) y z /(1+\varepsilon x+k) \\
& +\left(\gamma x y^{2} z\right) /(1+\eta x y)-b z .
\end{aligned}
$$

The variable $x$ stands for helper cells, $y$ denotes the replicating virus population, and $z$ stands for the CTL response. Virus replication is modelled as a simple density-dependent growth process. The helper cell population expands in response to antigenic stimulation, and delivers help to the CTL via both the classical and the CD4APC-CD8 pathway, as derived in this paper. Figure 4 shows simulations of this model for strong and weak helper cell responses. If the helper cell response is strong, the outcome of the model is suppression of virus load to low levels [Fig. 4(a)]. If we assume that extinction occurs below a threshold level of virus load, the CTL response clears the pathogen. At the beginning of the infectious process, the classical pathway induces faster CTL proliferation than the CD4-APC-CD8 pathway [Fig. 4(a)]. However, when virus load starts to decline to lower levels, the CD4-APC-CD8 pathway results in faster induction of CTL proliferation which results in the eventual clearance of the virus population [Fig. 4(a)]. On the other hand, if the helper cell response is weak, the CTL response fails to clear the infection resulting in persistent virus replication [Fig. 4(b)]. Initially, the classical pathway induces a relatively strong CTL responsiveness [Fig. 4(b)]. This is because at high loads, CTL proliferation resulting from the classical pathway becomes less dependent on the amount of help. However, immunological pressure is lost at lower 

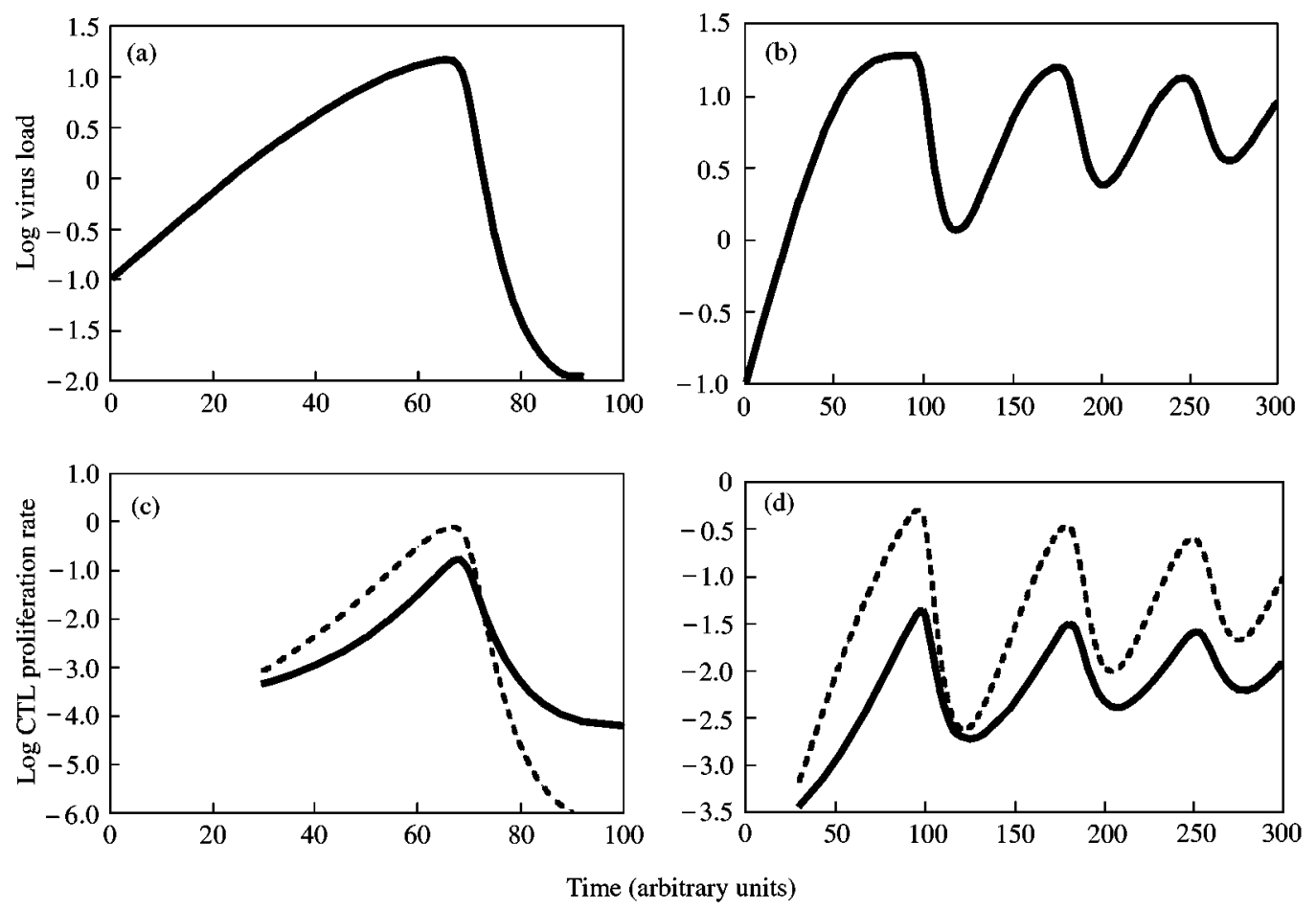

FIG. 4. Simulation showing the relevance of the classical pathway and the CD4-APC-CD8 pathway in the different stages of the infectious process. (a) Simulation assuming a relatively strong helper cell response, resulting in resolution of the infection. Initially, at high virus loads, the classical pathway is more efficient at inducing CTL proliferation. In the clearance phase of the infection, the CD4-APC-CD8 pathway is more efficient at maintaining CTL proliferation at low virus load. Note that we assume an extinction threshold for the virus, since the model is deterministic. (b) Simulation assuming a relatively weak helper cell response. Although initially the classical pathway induces relatively strong degrees of CTL proliferation at high virus loads, immunological pressure is lost at low loads, resulting in failure of the CTL response to resolve the infection. Parameters were chosen as follows: $\psi=0.05 ; r=0.1 ; \kappa=20 ; p=1 ; c=0.08 ; \varepsilon=1 ; \gamma=0.08 ; \eta=1 ; b=0.1$; for $(\mathrm{a}), \phi=0.02$; for (b) $\phi=0.007$ : (-- $)$ classical pathway; (—) CD4-APC-CD8 pathway.

virus loads resulting in failure to suppress the infection in the long term [Fig. 4(b)].

In summary, our mathematical models suggest different roles for the two pathways of help: the classical pathway is required to react quickly to high virus loads. Hence, the classical pathway could kick-start the CD8 cell response during the initial phases of the infectious process. On the other hand, the CD4-APC-CD8 pathway is required to ensure an efficient and fast response at low virus loads. Hence, the CD4-APC-CD8 pathway is important to ensure viral clearance following the acute phase of the infection.

\section{EXPERIMENTS WITH HELPER-DEFICIENT HOSTS}

In order to examine the role of help for the resolution of viral infections, experiments have been performed in hosts deficient in CD4 T cell responses. Of particular interest are experiments with lymphocytic choriomeningitis virus (LCMV) replicating in mice lacking major histocompatibility class II molecules (Thomsen et al., 1996). Such mice lack CD4 cell responses. During the acute phase of the infection, the mutant mice have a primary CD 8 cell response that is comparable to wild-type animals. Consequently, in both types of hosts, the initial viraemia is reduced below the level of detection. However, during the memory phase of the infection, class II deficient mice are characterized by the absence of specific CD8 cell responses, while wild-type mice show vigorous memory CD8 cell responses. Consequently, virus load starts to reemerge 30-60 days post-infection in mutant mice, while the virus remains undetectable for the time 
period of study in wild-type animals. The exact time it takes for virus load to re-emerge in mutant mice depends on the replication rate of the virus (Thomsen et al., 1996; Planz et al., 1997). Faster replicating strains of LCMV re-emerge faster than slower replicating strains.

In the context of our models, two explanations can be offered regarding the presence of normal CD8 cell responses in class II deficient mice in the acute phase of the infection. (i) As suggested by in vitro experiments (Deeths et al., 1999), CD8 cells might be able to produce IL-2 themselves and hence generate self-help for a limited number of divisions. (ii) LCMV has a tropism for APCs, such as dendritic cells and macrophages. Experiments suggest that infection of APCs might result in their activation, enabling them to deliver help in the absence of CD4 cell responses (Andreasen et al., 2000). Thus, during the acute phase of infection when virus load reaches high levels, many APCs are infected and become activated. This results in the delivery of help. As the CD8 cell responses reduce virus load, the number of infected APCs decreases, too. This results in loss of the help at low virus load, and consequently in re-emergence of viraemia and persistent infection. This is supported by a simulation of a model assuming that APCs are directly activated by the virus, resulting in the delivery of help (Fig. 5). In the simulation, virus load is initially reduced to low levels, followed by re-emergence of the virus. In accordance with experiments, the model further suggests that the time taken for virus reemergence correlates with the replication rate of the virus (Fig. 5).

The observation that viraemia re-emerges in class II deficient mice after a certain period of time implies that lack of help results in loss of immunological pressure at low virus loads. According to the model, this should be due to a defect in the CD4-APC-CD8 pathway. This is supported by further experimental evidence (Borrow et al., 1996, 1998; Thomsen et al., 1998). Mice deficient in CD40L and infected with LCMV show the same kind of behaviour as class II deficient mice infected with LCMV. Activation of APCs by CD4 cells is thought to require the binding of CD40L (on CD4 cells) with CD40 (on APCs). Hence, the CD4-APC-CD8 pathway is interrupted in $\mathrm{CD} 40 \mathrm{~L}-/-$ mice. On the other

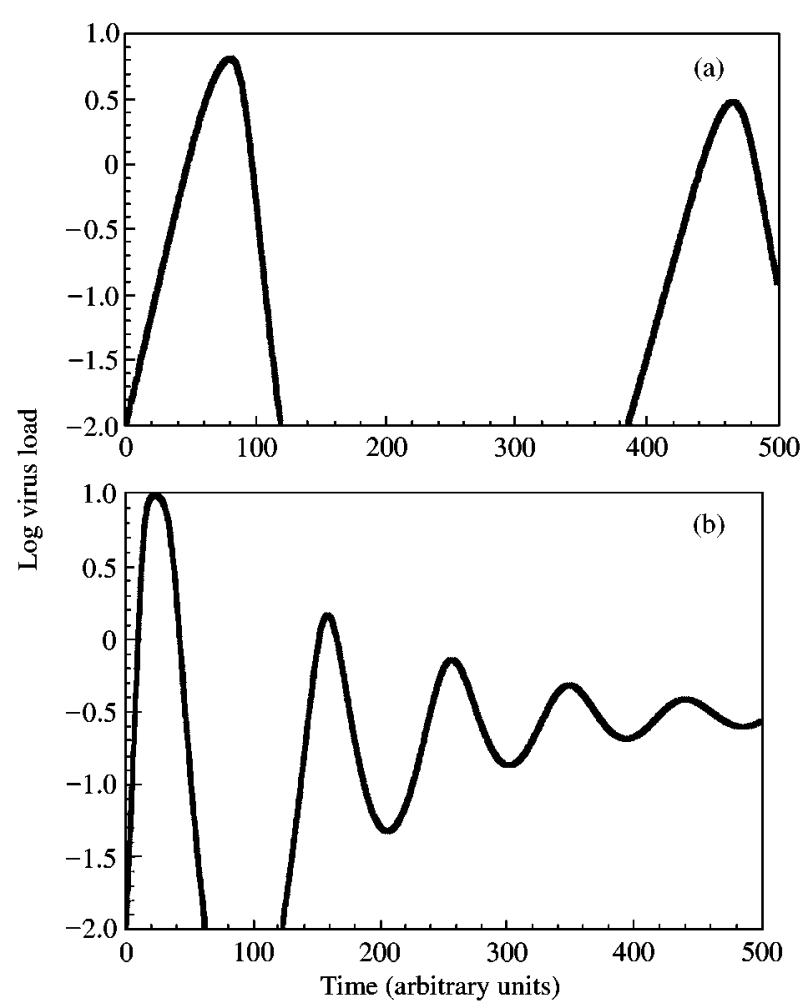

FIG. 5. Simulation of a model describing LCMV dynamics in helper-deficient hosts. It is assumed that LCMV infection results in the direct activation of APCs and the delivery of help, as derived in this paper. The model is given by the following pair of differential equations. $\mathrm{d} y / \mathrm{d} t=r y(1-y / \kappa)$ $-p y z, \mathrm{~d} z / \mathrm{d} t=(c k /(1+k)) y z-b z$, where $y$ represents the virus population and $z$ the CD8 cell population. (a) Simulation of a slowly replicating LCMV strain. (b) Simulation of a faster-replicating LCMV strain. Parameters were chosen as follows: $p=0.1 ; c=0.1 ; k=1 ; b=0.01 ; \kappa=10$; for (a) $r=0.1$; for (b) $r=0.5$.

hand, the classical pathway is unlikely to be deleted in these mutant mice. This interpretation also fits in with the observation that efficient CD8 cell mediated immunity can be restored in helper-deficient mice by cross-linking CD40 with antibodies (Ridge et al., 1998; Schoenberger et al., 1998).

\section{Implications for HIV Infection}

CD4 T cells are a major target for HIV replication. Clinical data from patients starting on anti-retroviral therapy have revealed that productively infected $\mathrm{T}$ cells have a relatively short half-life of about 2 days (Ho et al., 1995; Wei et al., 1995). Either viral cytotoxicity, and/or immune-mediated cell killing contributes to the 
death of the CD4 T cells (Klenerman et al., 1996). Hence, it is likely that during the initial stages of the infection, when HIV viraemia rises to high levels, most HIV-specific CD4 T cells become infected and killed. In support of this notion, clinical data from HIV-infected patients (Rosenberg et al., 2000) and experimental data from SIV-infected macaques (Lifson et al., 2000) have shown that significant levels of HIV-specific CD4 $\mathrm{T}$ cell proliferative responses are missing in typical infected individuals. This correlates with the generation of only weak and inefficient CD8 cell responses (Rosenberg et al., 2000). Figure 6 shows a simulation of an HIV-specific adaptation of the models derived in this paper. The model assumes that the target cells for the virus are immune cells, such as APCs and helper cells, and that infection of these cells induces immune impairment. For fast HIV replication, the model suggests that helper cell responses are significantly impaired, weakening the CTL response. This leads to persistent infection [Fig. 6(a)].

This is in contrast to long-term non-progressors, defined by the absence of pathology after 15 years or longer. Such patients are characterized by significant levels of HIV-specific CD4 cell proliferative responses, and high levels of specific CD8 cells despite very low virus loads (Harrer et al., 1996a, b; Rosenberg et al., 2000). According to the model (Fig. 6), this could occur if the replication rate of HIV is low. Slow virus spread results in only low amounts of helper cell impairment, and consequently, in stronger CTL responses. This leads to efficient suppression of HIV load to low levels [Fig. 6(b)].

Recently, therapy regimes have been discussed which can preserve specific CD4 cell responses, resulting in improved immunity (Wodarz et al., 1999; 2000; Lifson et al., 2000; Rosenberg et al., 2000; Lori et al., 2000). This is achieved most efficiently by treating early during the acute phase of the infection (Watson et al., 1997; Wodarz et al., 2000; Lifson et al., 2000; Rosenberg et al., 2000). Early therapy results in immune sensitization while preventing high degrees of HIV-induced helper cell impairment. This is thought to result in the availability of help and the generation of an efficient and sustained CD8 cell response that can control viral replication in the long term following the discontinuation of

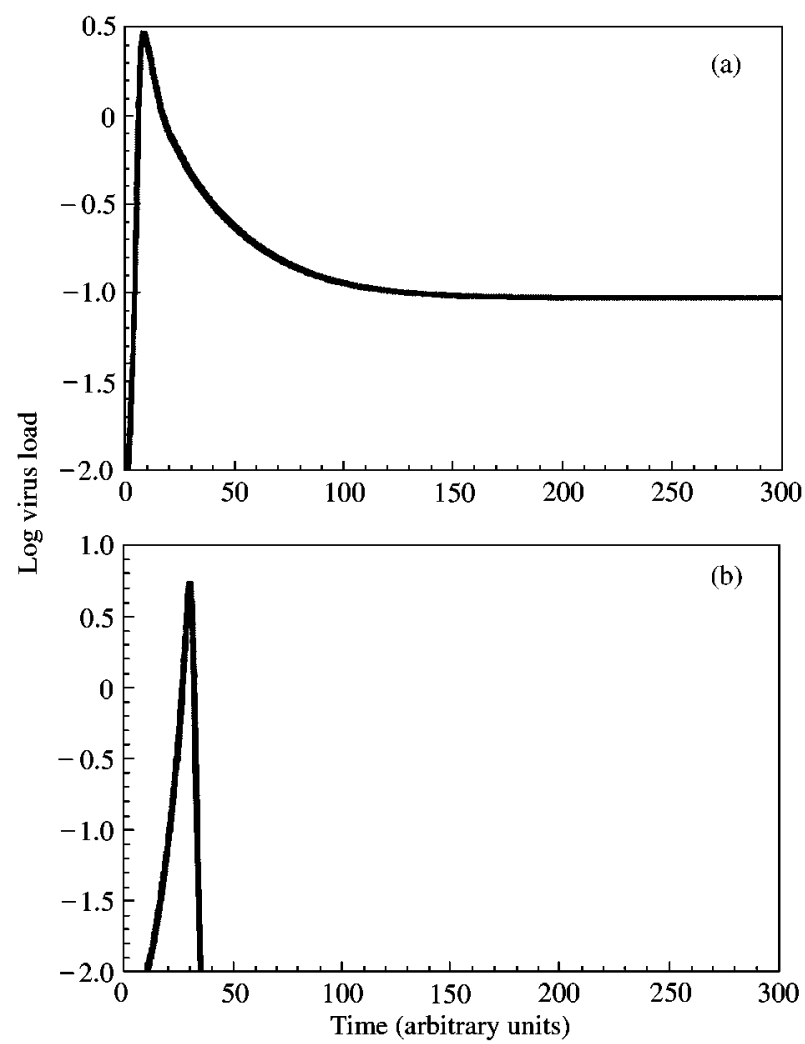

FIG. 6. Simulation of a model describing HIV dynamics, based on the models derived in this paper. The model is given by the following set of differential equations. $\mathrm{d} x / \mathrm{d} t=\lambda+\phi x y /(y+1)-\psi x-\beta x y, \mathrm{~d} y / \mathrm{d} t=\beta x y-a y-$ pyz, $\mathrm{d} z / \mathrm{d} t=c \varepsilon x y z /(1+\varepsilon x)+\left(\gamma x y^{2} z\right) /(1+\eta x y)-b z$. The variable $x$ represents the population of uninfected helper cells that are potential targets for HIV. The variable $y$ denotes infected cells, and $z$ denotes the CTL response. It is assumed that the population of helper cells are both produced at a given rate, and expand in response to antigenic stimulation. (Note that we assume that expansion saturates with high antigen concentrations in order to avoid a positive feedback between helper cell and virus growth.) Virus infection destroys the ability of these cells to deliver help. Hence, the degree of helper cell impairment correlates with the replication rate of the virus, $\beta$. (a) A fast-replicating HIV strain results in high degrees of helper cell impairment and weak CTL-mediated immunity. This leads to persistent infection. (b) A slowly replicating HIV strain results in less helper cell impairment and stronger CTL-mediated immunity. This can result in long-term control of the infection. Parameters were chosen as follows: $\phi=0.5 ; \psi=0.1 ; r=0.1$; $\kappa=20 ; p=1 ; c=0.1 ; \varepsilon=1 ; \gamma=0.1 ; \eta=1 ; b=0.01$; for (a) $\beta=0.7$; for (b) $\beta=0.1$.

therapy. Such effects can also be augmented and achieved by structured therapy interruptions (Wodarz et al., 1999; Rosenberg et al., 2000; Lori et al., 2000). Repeated therapy interruptions followed by reapplication of treatment have been shown to result in an increase in specific CD4 cell 
responses and in improved CD8 cell mediated control of HIV and SIV infection (Rosenberg et al., 2000; Lori et al., 2000). However, success of such therapy regimes depends on the repertoire of CD4 T cell specificities which are available and which can be induced. Hence, beyond a certain time threshold in the disease process, this repertoire will be extinct and significant levels of CD4 $\mathrm{T}$ cell responses cannot be induced anymore. How early treatment has to be started for interruptions to have a positive effect on the immune system is currently unclear. For further progress in HIV therapy, the exact nature of the defect in $\mathrm{T}$ cell help is an important question. Experimental evidence interpreted in the light of our models suggests that the defect lies in the CD4-APC-CD8 pathway. Clinical data from HIV-infected patients (Kalams et al., 1999) suggest that immunological pressure is weak at low virus loads. When virus load is reduced to low levels during drug therapy, the number and function of specific CD8 T cells significantly declines. On the other hand, long-term non-progressors, or individuals with improved immunological control following structured therapy interruptions are characterized by relatively high levels of CD 8 cells despite very low virus loads. The model further suggests that the classical pathway of help is unlikely to contribute to the long-term control of HIV, since it does not contribute to efficient CD8 cell mediated immunity at low virus loads. Indeed, it is likely that in typical HIV-infected patients, the classical pathway of help could be functional. Secretion of cytokines such as IL-2 is not so much restricted by the specificity of the CD4 T cells. Cytokines can be secreted by nonspecific CD4 $\mathrm{T}$ cells as a result of bystander activation. Thus, as long as the overall CD4 cell count is not depleted, cytokine secretion is likely to be functional. This can, in turn, maintain a specific CD8 cell response in the presence of relatively high degrees of antigenic stimulation throughout the chronic phase of the infection. Such a response can offer limited CD8 cell mediated control of HIV replication. These arguments have important implications for the design of immuno-therapeutic approaches with the aim of inducing long-term control of HIV infection. Administration of immuno-stimulatory cytokines, such as IL-2, is predicted to have little effect on the degree of virus control. While it would help to maintain CD8 cell responses throughout the chronic phase of the infection, these CD 8 cells would still be unable to provide efficient immunological pressure at low virus loads. Efficient CD8 cell responses at low loads, required for control, could be achieved only by enhancing the CD4-APC-CD8 cell pathway of help. In this respect, research should be aimed at directly inducing and activating the APCs, rather than at boosting specific CD4 T cell responses (as done with structured therapy interruptions). The reason is that during the disease process, the repertoire of CD4 $\mathrm{T}$ cells that has the potential to specifically react in response to HIV will become depleted. However, APCs are not specific and do not get significantly depleted during the course of the infection. Hence, as long as HIV-specific CD8 cell responses are around, direct therapeutic activation of APCs could result in the delivery of help which could result in improved control of the infection. Such direct, CD4 cell-independent induction of APCs could result in improved immunity even during later stages of the disease, as long as HIV-specific CD8 $\mathrm{T}$ cell responses are still available.

\section{AN EXPERIMENTAL TEST}

In this paper we have used mathematical models in order to analyse the role of CD4 cell help in the context of CD8 cell responses. We constructed models describing both the classical pathway of help, and the CD4-APC-CD8 pathway. The models suggested that the classical pathway is more efficient at reacting in response to high antigen loads, while the CD4-APC-CD8 pathway is more efficient at exerting strong CD8 cell mediated activity at low antigen loads. This not only has important conceptual implications for understanding the role of help in the generation of $\mathrm{CD} 8$ cell responses, but also provides important new insights into possible immunotherapeutic strategies aimed at controlling immuno-suppressive infections such as HIV. The models also suggest a simple experimental test in order to distinguish the importance of the two pathways of help in infections. The rate of CD8 cell proliferation should be measured as a function of antigen load (Fig. 3). If the 
CD4-APC-CD8 pathway is more important, then CD8 cell proliferation should be a linear function of load. On the other hand, if the classical pathway is more important, then CD8 cell proliferation is not a linear function of antigen load, and for low antigen load this relation is quadratic. It might also be interesting to test this relationship separately for low and high antigen loads, since the models suggest that the relative importance of the two pathways of help might depend on the level of antigen load against which the CD8 cells have to react (Fig. 6).

VAAJ gratefully acknowledges financial support from the Wellcome Trust, Grant No. 051319.

\section{REFERENCES}

Andreasen, S. O., Christensen, J. E., Marker, O. \& Thomsen, A. R. (2000). Role of CD40 ligand and CD28 in induction and maintenance of antiviral CD8 + effector $\mathrm{T}$ cell responses. J. Immunol. 164, 3689-3697.

Borghans, J. A., TAAms, L. S., WaUben, M. H. \& DE Boer, R. J. (1999). Competition for antigenic sites during T cell proliferation: a mathematical interpretation of in vitro data. Proc. Natl Acad. Sci. U.S.A. 96, 10 782-10 787.

Borrow, P., Tishon, A., LeE, S., XU, J., Grewal, I. S., Oldstone, M. B. \& Flavell, R. A. (1996). CD40L-deficient mice show deficits in antiviral immunity and have an impaired memory CD8 + CTL response. J. Exp. Med. 183, 2129-2142.

Borrow, P., Tough, D. F., Eto, D., Tishon, A., Grewal, I. S., Sprent, J., Flavell, R. A. \& Oldstone, M. B. (1998). CD40 ligand-mediated interactions are involved in the generation of memory $\mathrm{CD} 8(+)$ cytotoxic T lymphocytes (CTL) but are not required for the maintenance of CTL memory following virus infection. J. Virol. 72, 7440-7449.

Christensen, J. P., Cardin, R. D., Branum, K. C. \& DoherTY, P. C. (1999). CD4(+ ) T cell-mediated control of a gamma-herpesvirus in $\mathrm{B}$ cell-deficient mice is mediated by IFN-gamma. Proc. Natl Acad. Sci. U.S.A. 96, 5135-5140.

DeBoer, R. J. \& Perelson, A. S. (1994). T cell repertoires and competitive exclusion. J. theor. Biol. 169, 375-390.

Deeths, M. J., KedL, R. M. \& Mescher, M. F. (1999). $\mathrm{CD} 8+\mathrm{T}$ cells become nonresponsive (anergic) following activation in the presence of costimulation. J. Immunol. 163, 102-110.

Fishman, M. A. \& Perelson, A. S. (1993). Modeling T cellantigen presenting cell interactions. J. theor. Biol. 160, 311-342.

Harrer, T., Harrer, E., Kalams, S. A., Barbosa, P., Trocha, A., Johnson, R. P., Elbeik, T., FeinberG, M. B., Buchionder, S. P. \& Walker, B. D. (1996a). Cytotoxic T lymphocytes in asymptomatic long-term nonprogressing HIV-1 infection. Breadth and specificity of the response and relation to in vivo viral quasispecies in a person with prolonged infection and low viral load. J. Immunol. 156, 2616-2623.
Harrer, T., Harrer, E., Kalams, S. A., Elbeik, T., StapRAns, S. I., FeinberG, M. B., CaO, Y., Ho, D. D., Yilma, T., Caliendo, A. M., Johnson, R. P., Buchbinder, S. P. \& Walker, B. D. (1996b). Strong cytotoxic T cell and weak neutralizing antibody responses in a subset of persons with stable nonprogressing HIV type 1 infection. AIDS Res. Hum. Retroviruses 12, 585-592.

Ho, D. D., Neumann, A. U., Perelson, A. S., Chen, W., LEONARD, J. M. \& MARKOWITZ, M. (1995). Rapid turnover of plasma virions and Cd4 lymphocytes in HIV-1 infection. Nature 373, 123-126.

Jeffery, K. J., Usuku, K., Hall, S. E., Matsumoto, W., Taylor, G. P., Procter, J., Bunce, M., OGg, G. S., Welsh, K. I., Weber, J. N., Lloyd, A. L., NowaK, M. A., NAGai, M., KodAMA, D., IzUmo, S., Osame, M. \& BANGHAM, C. R. (1999). HLA alleles determine human T-lymphotropic virus-I (HTLV-I) proviral load and the risk of HTLV-I-associated myelopathy [in process citation]. Proc. Natl Acad. Sci. U.S.A 96, 3848-3853.

KaGi, D., LedermanN, B., Burki, K., Zinkernagel, R. M. \& Hengartner, H. (1996). Molecular mechanisms of lymphocyte-mediated cytotoxicity and their role in immunological protection and pathogenesis in vivo. Annu. Rev. Immunol. 14, 207-232.

KALAMS, S. A. \& WALKER, B. D. (1998). The critical need for CD4 help in maintaining effective cytotoxic T lymphocyte responses [comment]. J. Exp. Med. 188, 2199-2204.

Kalams, S. A., Goulder, P. J., Shea, A. K., Jones, N. G., Trocha, A. K., OGg, G. S. \& Walker, B. D. (1999). Levels of human immunodeficiency virus type 1-specific cytotoxic T-lymphocyte effector and memory responses decline after suppression of viremia with highly active antiretroviral therapy. J. Virol. 73, 6721-6728.

Klenerman, P., Phillips, R. E., Rinaldo, C. R., Wahl, L. M., OgG, G., May, R. M., McMichael, A. J. \& NowAK, M. A. (1996). Cytotoxic T lymphocytes and viral turnover in HIV type 1 infection. Proc. Natl Acad. Sci. U.S.A. 93, $15323-15328$.

Lifson, J. D., Rossio, J. L., Arnaout, R., Li, L., Parks, T. L., Schneider, D. M., Kiser, R. F., CoAlter, V. J., Walsh, G., Imming, R., Fischer, B., FlynN, B. M., NowaK, M. A. \& WodArz, D. (2000). Containment of SIV infection: cellular immune responses and protection from rechallenge following transient post-inoculation antiretroviral treatment. J. Virol. 74, 2584-2593.

LORI, F., Lewis, M. G., XU, J., VArGa, G., ZinN JR., D. E., Crabbs, C., Wagner, W., Greenhouse, J., Silvera, P., Yalley-OGunro, J., Tinelli, C. \& Lisziewicz, J. (2000). Control of SIV rebound through structured treatment interruptions during early infection. Science 290, 1591-1593.

NowaK, M. A. \& Bangham, C. R. (1996). Population dynamics of immune responses to persistent viruses. Science 272, 74-79.

Planz, O., Ehl, S., Furrer, E., Horvath, E., Brundler, M. A., Hengartner, H. \& Zinkernagel, R. M. (1997). A critical role for neutralizing-antibody-producing B cells, CD4 $(+) \mathrm{T}$ cells, and interferons in persistent and acute infections of mice with lymphocytic choriomeningitis virus: implications for adoptive immunotherapy of virus carriers. Proc. Natl Acad. Sci. U.S.A. 94, 6874-6879.

Ridge, J. P., Di RosA, F. \& MAtzinger, P. (1998). A conditioned dendritic cell can be a temporal bridge between a CD4 + T-helper and a T-killer cell [see comments]. Nature 393, 474-478. 
Rosenberg, E. S., Billingsley, J. M., Caliendo, A. M., Boswell, S. L., SAX, P. E., Kalams, S. A. \& Walker, B. D. (1997). Vigorous HIV-1-specific CD4 + T cell responses associated with control of viremia [see comments]. Science 278, 1447-1450.

Rosenberg, E. S., Altfeld, M., Poon, S. H., Phillips, M. N., Wilkes, B. M., Eldridge, R. L., Robbins, G. K., D'Aquila, R. T., Goulder, P. J. \& Walker, B. D. (2000). Immune control of HIV-1 after early treatment of acute infection [in process citation]. Nature 407, 523-526.

Schmitz, J. E., Kuroda, M. J., Santra, S., Sasseville, V. G., Simon, M. A., Lifton, M. A., RACZ, P., TEnNer-RACZ, K., Dalesandro, M., Scallon, B. J., Ghrayeb, J., ForMAN, M. A., Montefiori, D. C., Rieber, E. P., LetVin, N. L. \& REIMANN, K. A. (1999). Control of viremia in simian immunodeficiency virus infection by $\mathrm{CD} 8(+)$ lymphocytes [in process citation]. Science 283, 857-860.

Schoenberger, S. P., Toes, R. E., VAn Der VoOrt, E. I., OffringA, R. \& Melief, C. J. (1998). T-cell help for cytotoxic T lymphocytes is mediated by CD40-CD40L interactions [see comments]. Nature 393, 480-483.

Thomsen, A. R., Johansen, J., MARKer, O. \& ChristenSEN, J. P. (1996). Exhaustion of CTL memory and recrudescence of viremia in lymphocytic choriomeningitis virusinfected MHC class II-deficient mice and B cell- deficient mice. J. Immunol. 157, 3074-3080.

Thomsen, A. R., Nansen, A., Christensen, J. P., Andreasen, S. O. \& Marker, O. (1998). CD40 ligand is pivotal to efficient control of virus replication in mice infected with lymphocytic choriomeningitis virus. J. Immunol. 161, 4583-4590.

Watson, A., McClure, J., Ranchalis, J., Scheibel, M., SChMidT, A., KenNedy, B., Morton, W. R., Haigwood, N. L. \& HU, S. L. (1997). Early postinfection antiviral treatment reduces viral load and prevents CD4 + cell decline in HIV type 2-infected macaques. AIDS Res. Hum. Retroviruses 13, 1375-1381.

Wei, X. P., Ghosh, S. K., TAYlor, M. E., Johnson, V. A., Emini, E. A., Deutsch, P., Lifson, J. D., Bonhoeffer, S., NowaK, M. A., HaHn, B. H., SAAG, M. S. \& SHAw, G. M. (1995). Viral dynamics in human-immunodeficiencyvirus type-1 infection. Nature 373, 117-122.

WODARZ, D. \& NowAK, M. A. (1999). Specific therapy regimes could lead to long-term control of HIV. Proc. Natl Acad. Sci. U.S.A. 96, $14464-14469$.

WoDARZ, D. \& NowAK, M. A. (2000). Immune responses and viral phenotype: do replication rate and cytopathogenicity influence virus load? J. Theor. Med. 2, $113-127$.

WodARz, D., NowaK, M. A. \& BAngham, C. R. M. (1999). The dynamics of HTLV-1 and the CTL response. Immunol. Today 20, 220-227.

Wodarz, D., Arnaout, R. A., NowaK, M. A. \& Lifson, J. D. (2000). Transient antiretroviral treatment during acute SIV infection facilitates long-term control of the virus. Phil. Trans. Roy. Soc. Lond. B. 355, 1021-1029. 